\title{
IMPACTO DA DISPERSÃO, AUTOMODULAÇÃO DE FASE, MODULAÇÃO DE FASE CRUZADA E DESCASAMENTO DE VELOCIDADE DE GRUPO NA PROPAGAÇÃO DE TRENS DE PULSOS EM FIBRA ÓPTICA MONOMODO
}

\author{
Paula Brandão Harboe ${ }^{1}$ \\ José Rodolfo Souza ${ }^{2}$ \\ David Santos Godoy
}

\begin{abstract}
Resumo: $\mathrm{O}$ presente trabalho investiga o impacto dos mecanismos de dispersão de velocidade de grupo (GVD), automodulação de fase (SPM), modulação de fase cruzada (XPM) e descasamento de velocidade de grupo (walk-off) na propagação de trens de pulsos em fibra óptica monomodo. O modelo matemático é baseado em um conjunto de equações não-lineares de Schrödinger (NLSE) acopladas que são resolvidas numericamente com o Método da Propagação de Feixes (BPM). Considerando um sistema multiplexado por divisão em comprimento de onda (WDM), o modelo permite avaliar como o envelope e o espectro de pulsos de diferentes formatos são modificados pelos mecanismos acima citados, para diversas condiçóes iniciais de propagação. Os resultados numéricos obtidos estão em perfeita concordância com os disponíveis na literatura e mostram a versatilidade e robustez do modelo.
\end{abstract}

Palavras-Chave: Sistemas WDM, Dispersão de Velocidade de Grupo (GVD), Não-linearidades Ópticas (SPM, XPM) e Walk-Off.

\begin{abstract}
This paper investigates how the mechanisms of group-velocity dispersion (GVD), self-phase modulation (SPM), cross-phase modulation, and group-velocity walk-off impact the propagation of pulse trains along monomode optical fiber. The mathematical model is based on a set of of coupled nonlinear Schrödinger equations (NLSE), solved numerically with the Beam Propagation Method (BPM). Designed for WDM systems, the model allows for the evaluation of the alteration suffered by the waveform, and spectrum of pulses of different shapes, under different initial conditions of propagation. The numerical results obtained are in perfect accordance with others in the literature, and confirm the versatility, as well as the robustness of the model.
\end{abstract}

Keywords: WDM Systems, Group-Velocity Dispersion (GVD), Fiber Nonlinearities (SPM, XPM), Pulse walk-off.

\section{INTRODUÇÃO}

Sistemas DWDM (Dense Wavelength Division Multiplexing) com elevado número de canais finamente espaçados possibilitam a exploração da ampla largura de banda oferecida por fibras ópticas, aumentando a capacidade de redes já existentes e permitindo a instalação de novas plantas.
Entretanto, as não-linearidades ópticas - SPM (Self-Phase Modulation - automodulação de fase), XPM (Cross-Phase Modulation - modulação de fase cruzada) e FWM (Four-Wave Mixing - mistura de quatro ondas) - em conjunto com a dispersão de velocidade de grupo (GVD - GroupVelocity Dispersion), provocam interaçóes entre os canais, resultando em sérias distorções dos sinais e

\footnotetext{
1 Departamento de Engenharia de Telecomunicações, Universidade Federal Fluminense, paula@telecom.uff.br

2 Departamento de Eletrônica e Telecomunicaçōes, Universidade do Estado do Rio de Janeiro, jrsouza@uerj.br

3 Departamento de Engenharia de Telecomunicações, Universidade Federal Fluminense, davidsgodoy@yahoo.com.br
} 
na degradação do desempenho dos sistemas (WU; WAY, 2004).

Os efeitos SPM, XPM e FWM têm origem na refração não-linear (efeito Kerr), um fenômeno que se caracteriza pela dependência entre o índice de refração e a intensidade óptica. SPM é responsável pela modulação da fase do próprio pulso, o que provoca um desvio da freqüência instantânea em relação à freqüência central. Este efeito é comumente chamado de chirp de freqüência. No caso de XPM, o mesmo efeito é observado, mas agora provocado por pulsos vizinhos. Por sua vez, contribuições significativas de FWM ocorrem quando a condição de casamento de fase é satisfeita, resultando em um batimento entre canais e na geração de novas frequiências ópticas. $\mathrm{O}$ mecanismo de FWM pode ser minimizado através de uma escolha adequada do espaçamento entre canais, da potência de entrada por canal, do tipo de fibra óptica empregado, do posicionamento dos canais em relação ao comprimento de onda do zero de dispersão da fibra, assim como da taxa e distância de transmissão.

A propagação de sinais luminosos em fibra óptica é governada pela bem conhecida Equação Não-Linear de Schrödinger (NLSE) (AGRAWAL, 2006). Na maioria dos casos estudados, esta equação não admite solução analítica, exigindo o emprego de aproximaçôes numéricas, como, por exemplo, o Método da Propagação de Feixes (BPM) (AGRAWAL, 2006).

A análise e otimização de desempenho de sistemas WDM modernos requerem, portanto, o desenvolvimento de ferramentas numéricas (pacotes de software) robustas, eficientes do ponto de vista computacional e versáteis, capazes de simular diversas situações de interesse prático, a custos relativamente baixos.

Usualmente, a implementação de modelos numéricos completos consome elevado tempo computacional. Estudos mostram, ainda, que em torno de $60 \%$ do tempo de simulação são despendidos com cálculos realizados na fibra óptica, o que reforça a importância de se desenvolver e disponibilizar ferramentas eficientes (ROUDAS, 2000).

A literatura indica que, por exemplo, para uma correta avaliação de FWM, o passo de discretização usado no BPM deve ser consideravelmente menor que o passo usado para a modelagem de XPM (LEIBRICH; ROSENKRANZ, 2003).

Uma abordagem interessante e vantajosa do ponto de vista computacional e proposta pelos au- tores, consiste em considerar a propagação de pulsos sob os efeitos de XPM e SPM separadamente dos de FWM. Para isto, situações que promovam a condição de casamento de fase, inerentemente satisfeita por XPM, e necessária para a eficiente geração de FWM, devem ser identificadas e evitadas.

Os autores, em trabalhos anteriores (SOUZA; HARBOE, 2003) e recentes (HARBOE; SILVA; SOUZA, 2008a-2008c), investigaram cuidadosamente as penalidades impostas por FWM em sistemas WDM utilizando fibras G.652 (SMF - fibra monomodo padrão), G.653 (DSF - fibra de dispersão deslocada) e G.655 (NZDSF - fibra de dispersão deslocada não-nula), considerando espaçamentos uniforme $(100,50,25,12,5$ e 6,25 $\mathrm{GHz}$ ) e não-uniforme entre canais. Os numerosos resultados mostram que a redução dos efeitos deletérios de FWM e a manutenção de valores aceitáveis para a relação sinal-ruído (SNR) do sistema dependem, em parte da: i) alocação dos canais fora da região do zero de dispersão da fibra DSF; ii) utilização de fibra NZDSF, cujo parâmetro de dispersão é pequeno, mas não nulo na região de $1550 \mathrm{~nm}$ e iii) utilização de fibra SMF (largamente instalada em todo o mundo), que possui elevada dispersão na região de $1550 \mathrm{~nm}$. As situações investigadas deixaram claro, também, que um esquema de alocação de canais com espaçamento não uniforme, embora complexo, é praticamente obrigatório em sistemas empregando fibra DSF (HARBOE; SILVA; SOUZA, 2008b).

$\mathrm{O}$ presente trabalho investiga as alterações nas formas temporal (envelope) e espectral de pulsos co-propagantes em fibra óptica monomodo sob o efeito conjunto dos mecanismos de dispersão (GVD), não-linearidades (SPM e XPM) e descasamento de velocidade de grupo dos pulsos (walk-off). O modelo matemático é baseado na solução numérica de um conjunto de NLSE acopladas sob diferentes condições iniciais de propagação. O mecanismo de FWM não é considerado nesta análise.

No entendimento dos autores, o "saberfazer" é fundamental para o desenvolvimento de soluções que atendam satisfatoriamente às necessidades de projeto. Além disso, o desenvolvimento de pacotes de software leves permite sua utilização em ambiente de sala de aula, complementando e exemplificando as explicações teóricas em disciplinas na área de Comunicações Ópticas.

O trabalho está organizado da seguin- 
te maneira: inicialmente, na Seção 2, o modelo matemático é apresentado e a influência de cada termo no conjunto de equações acopladas é destacada. Três escalas de comprimento, denominadas comprimento de dispersão $\mathrm{L}_{\mathrm{D}}$, comprimento de não-linearidade $\mathrm{L}_{\mathrm{NL}}$ e comprimento de walkoff de pulsos $\mathrm{L}_{\mathrm{w}}$, são introduzidas para enfatizar a importância relativa dos efeitos associados. Um simulador numérico é, então, desenvolvido usando a linguagem FORTRAN 77. Em seguida, na Seção 3, dois casos distintos são investigados. Primeiramente, os efeitos da dispersão (GVD) são desprezados e o alargamento espectral e deslocamento de freqüência dos pulsos são observados sob o efeito conjunto das não-linearidades e do walk-off. Em seguida, a dispersão é incluída e as alteraçōes nas formas temporal (envelope) e espectral dos pulsos são investigadas e comparadas com a situação anterior. Neste caso, observam-se os fenômenos de quebra de onda óptica (optical wave breaking) e da geração de pulsos escuros a partir de onda contínua (CW). Este último vem sendo utilizado, no contexto de redes ópticas de alta velocidade, como conversor de comprimento de onda. Os resultados numéricos são discutidos e estão em excelente concordância com outros encontrados na literatura. Situações envolvendo o acoplamento não-linear induzido por SPM e $\mathrm{XPM}$, tais como as compressões temporal e espectral de pulsos, podem ser facilmente analisadas utilizando o algoritmo desenvolvido e revelam a versatilidade e robustez da ferramenta numérica. Para finalizar, na Seção 4 são apresentadas as conclusões do trabalho.

\section{FORMALISMO MATEMÁTICO}

A propagação simultânea de pulsos em fibra óptica monomodo, dispersiva, não-linear e com perdas é governada, em determinadas condiçôes, pela NSLE. A equação diferencial que descreve a evolução do envelope complexo $A_{k}(z, T)$ do késimo canal é dada por (HARBOE; GODOY; SOUZA, 2006):

$$
\begin{gathered}
\frac{\partial A_{k}}{\partial z}+d_{k} \frac{\partial A_{k}}{\partial T}+\frac{j}{2} \beta_{2 k} \frac{\partial^{2} A_{k}}{\partial T^{2}}-\frac{\beta_{3 k}}{6} \frac{\partial^{3} A_{k}}{\partial T^{3}}+\frac{\alpha}{2} A_{k}= \\
j \gamma_{k}\left(\left|A_{k}\right|^{2}+2 \sum_{i(i \neq k)}\left|A_{i}\right|^{2}\right) A_{k}
\end{gathered}
$$

Por simplicidade, a obtenção da equação (1) foi baseada nas hipóteses de que o modo fundamental $\mathrm{HE}_{11}$ é linearmente polarizado e que o campo óptico mantém sua polarização durante a propagação. Dessa forma, efeitos de birrefringência são ignorados e a solução da equação (1) é escalar.

Dois tipos de pulsos são considerados neste trabalho: pulsos claros e pulsos escuros. Pulsos claros são caracterizados por um perfil de alta intensidade em um fundo de baixa intensidade. Pulsos escuros, por sua vez, têm perfil de intensidade que consiste de uma depressão em um fundo de intensidade uniforme. A Figura 1 ilustra estes dois conceitos.

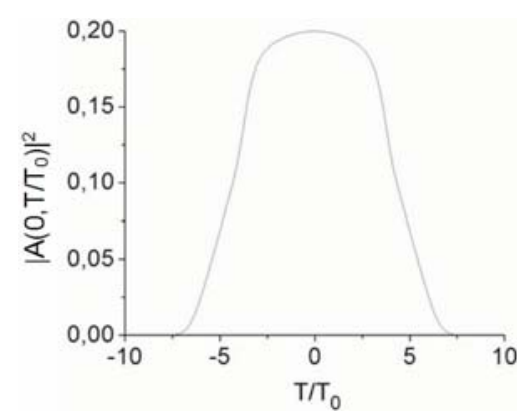

(a)

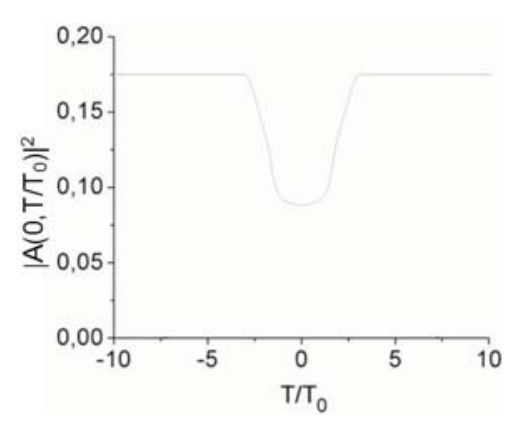

(b)

Figura 1: (a) Pulso claro, (b) pulso escuro. T0 é a largura do pulso.

$\mathrm{Na}$ equação (1), o referencial temporal $\mathrm{T}=$ $\mathrm{t}-\mathrm{z} / \mathrm{v}_{\text {gref }}$ se move ao longo do eixo $\mathrm{z}$ com a velocidade de grupo $\mathrm{v}_{\text {gref }}$ de um canal de referência escolhido arbitrariamente. $\mathrm{O}$ envelope do k-ésimo canal $A_{k}(z, T)$, de variação lenta, se move com velocidade de grupo $\mathrm{v}_{\mathrm{gk}}=1 / \beta_{1 \mathrm{k}}$. O efeito de GVD é representado pelos parâmetros $\beta_{2 \mathrm{k}}$ e $\beta_{3 \mathrm{k}}$. A interação entre o canal k e o canal de referência é determinada pelo descasamento de velocidade de grupo $\mathrm{d}_{\mathrm{k}}=\mathrm{v}_{\mathrm{gk}}^{-1}-\mathrm{v}_{\text {gref }}^{-1}$, fenômeno conhecido como 
walk-off. Os efeitos não-lineares SPM e XPM são representados, respectivamente, pelo primeiro e segundo termos no lado direito da equação (1), que indicam que XPM é duas vezes mais eficiente que SPM para uma mesma intensidade. $\mathrm{O}$ coeficiente não-linear $\gamma_{\mathrm{k}}=2 \pi \mathrm{n}_{2} / \lambda_{\mathrm{k}} \mathrm{A}_{\mathrm{eff}}$, onde $\mathrm{n}_{2}$ é o coeficiente de índice de refração não-linear da fibra, $\lambda_{\mathrm{k}}$ é o comprimento de onda do k-ésimo canal e $\mathrm{A}_{\text {eff }}$ é a área efetiva do núcleo da fibra. $\mathrm{O}$ parâmetro $\alpha$ representa a atenuação da fibra, e $\mathrm{j}=\sqrt{-1}$.

No presente trabalho, considera-se, primeiramente, a propagação de pulsos em fibra monomodo padrão (SMF) na região visível do espectro eletromagnético e, em seguida, em fibra de dispersão deslocada (DSF) na janela de $1550 \mathrm{~nm}$. Nas duas situações a propagação ocorre em região afastada do zero de dispersão da fibra, onde os efeitos de FWM podem ser desprezados devido ao elevado descasamento de fase.

Tipicamente, na região visível do espectro eletromagnético (região de dispersão normal), $\mathrm{A}_{\text {eff }}=$ $10-20 \mu \mathrm{m}^{2}, \gamma=20-30$ (W.km) ${ }^{-1}$ e $\beta_{2}$ é positivo. $\mathrm{Na}$ região infravermelha (região de dispersão anômala), $\mathrm{A}_{\text {eff }}=50-80 \mu \mathrm{m}^{2}, \gamma \approx 3$ (W.km) ${ }^{-1}$ e $\beta_{2}$ é negativo.

$\mathrm{O}$ parâmetro de dispersão $\mathrm{D}_{\mathrm{k}}$ para as fibras SMF e DSF é escrito como nas equações (2a) e (2b), respectivamente (HARBOE; GODOY; SOUZA, 2006):

$$
\begin{gathered}
\mathrm{D}_{\mathrm{k}}=\frac{\mathrm{S}_{\mathrm{k}}}{4}\left(\lambda_{\mathrm{k}}-\frac{\lambda_{0}^{4}}{\lambda_{\mathrm{k}}^{3}}\right) \\
\mathrm{D}_{\mathrm{k}}=\mathrm{S}_{\mathrm{k}}\left(\lambda_{\mathrm{k}}-\lambda_{0}\right)
\end{gathered}
$$

e se relaciona com os parâmetros $\beta_{1 \mathrm{k}}, \beta_{2 \mathrm{k}}$ e $\beta_{3 \mathrm{k}}$ através de (HARBOE; GODOY; SOUZA, 2006):

$$
\begin{gathered}
\beta_{1 \mathrm{k}}=\int \mathrm{D}_{\mathrm{k}} \mathrm{d} \lambda(3 \mathrm{a}) \\
\beta_{2 \mathrm{k}}=-\frac{\lambda_{\mathrm{k}}^{2}}{2 \pi \mathrm{c}} \mathrm{D}_{\mathrm{k}}(3 \mathrm{~b}) \\
\beta_{3 \mathrm{k}}=\frac{\lambda_{\mathrm{k}}^{3}}{(2 \pi \mathrm{c})^{2}}\left(2 \mathrm{D}_{\mathrm{k}}+\lambda_{\mathrm{k}} \mathrm{S}_{\mathrm{k}}\right)(3 \mathrm{c})
\end{gathered}
$$

Nas equações (2)-(3), $\mathrm{S}_{\mathrm{k}}=\mathrm{dD}_{\mathrm{k}} / \mathrm{d} \lambda$ é a inclinação (slope) da curva de dispersão, c é a velocidade da luz no vácuo e $\lambda_{0}$ é o comprimento de onda de zero de dispersão da fibra.
Após alguma manipulação algébrica, o descasamento de velocidade de grupo $\mathrm{d}_{\mathrm{k}}$ é calculado para as fibras SMF e DSF como nas equações (4a) e (4b), respectivamente (GODOY, 2006):

$$
\begin{gathered}
\mathrm{d}_{\mathrm{k}}=\frac{\mathrm{S}_{\mathrm{k}}}{8}\left(\lambda_{\mathrm{k}}^{2}-\lambda_{\text {ref }}^{2}\right)\left(1-\frac{\lambda_{0}^{4}}{\lambda_{\mathrm{k}}^{2} \lambda_{\text {ref }}^{2}}\right) \\
\mathrm{d}_{\mathrm{k}}=\mathrm{S}_{\mathrm{k}}\left(\lambda_{\mathrm{k}}-\lambda_{\text {ref }}\right)\left(\frac{\lambda_{\mathrm{k}}-\lambda_{\text {ref }}}{2}-\lambda_{0}\right)
\end{gathered}
$$

Nas equações (4), $\lambda_{\text {ref }}$ é o comprimento de onda do canal de referência. As seguintes escalas de comprimento são convenientemente introduzidas (HARBOE; GODOY; SOUZA, 2006):

$$
\begin{aligned}
\mathrm{L}_{\mathrm{D}}^{(\mathrm{k})} & =\mathrm{T}_{0 \mathrm{k}}^{2} /\left|\beta_{2 \mathrm{k}}\right| \\
\mathrm{L}_{\mathrm{NL}}^{(\mathrm{k})} & =1 / \gamma_{\mathrm{k}} \mathrm{P}_{\mathrm{k}} \\
\mathrm{L}_{\mathrm{W}}^{(\mathrm{k})} & =\mathrm{T}_{0 \mathrm{k}} /\left|\mathrm{d}_{\mathrm{k}}\right|
\end{aligned}
$$

Nas equações (5), $\mathrm{T}_{0 \mathrm{k}}$ e $\mathrm{P}_{\mathrm{k}}$ correspondem, respectivamente, à largura e potência de pico dos pulsos do k-ésimo canal. A solução da equação (1) é obtida de forma mais simples e eficiente comparando-se os comprimentos de dispersão $\mathrm{L}_{\mathrm{D}}$, de não-linearidade $\mathrm{L}_{\mathrm{NL}}$ e de walk-off $\mathrm{L}_{\mathrm{W}}$ com o comprimento total do enlace L. Em casos onde $\beta_{2 \mathrm{k}}$ é nulo (operação no zero de dispersão da fibra) ou na propagação de pulsos ultracurtos, os efeitos dispersivos de mais baixa ordem são governados por $\beta_{3 \mathrm{k}}$ e o comprimento de dispersão é reescrito como $\mathrm{L}_{\mathrm{D}}^{(\mathrm{k})}=\mathrm{T}_{0 \mathrm{k}}^{3} /\left|\beta_{3 \mathrm{k}}\right|$.

As equações (1)-(5) fornecem o formalismo matemático necessário para o estudo de casos apresentado a seguir.

\section{DISCUSSÃO DE RESULTADOS}

O modelo matemático e o simulador numérico desenvolvidos permitem analisar as alteraçôes temporal e espectral de trens de pulsos, com número arbitrário de pulsos e sob variadas condições iniciais de propagação. Por questão de simplicidade e visando um melhor entendimento 
dos fenômenos físicos associados, os casos seguintes consideram a propagação de dois pulsos apenas. As situações de ausência e presença de GVD são tratadas e ilustradas separadamente. A configuração pump/probe é adotada, ou seja, a potência de pico de um dos pulsos (pump) é considerada muito maior que a do outro (probe) de forma a realçar uma das não-linearidades ópticas (SPM ou XPM). Ainda, para melhor visualização, a atenuação da fibra é desprezada $(\alpha=0)$.

Simulações considerando um maior número de pulsos também foram realizadas e, quando pertinente, os principais resultados são comentados.

\subsection{XPM NA AUSÊNCIA DE GVD}

Inicialmente, analisa-se a interação entre pulsos gaussianos de luz verde $(530 \mathrm{~nm}, 100 \mathrm{~mW})$ e luz laranja $(630 \mathrm{~nm}, 100 \mathrm{~W})$ que se propagam em uma fibra monomodo padrão SMF $\left(\lambda_{0}=1310\right.$ $\mathrm{nm})$ no regime de dispersão normal. $\mathrm{O}$ pulso verde (pulso 1) é tomado como referência e sua potência de pico é muito menor que a do pulso laranja (pulso 2). Nessa análise, portanto, o efeito de XPM é acentuado no pulso verde, enquanto que o efeito de SPM é mais significativo no pulso laranja. Considerando pulsos com largura $\mathrm{T}_{0}=10 \mathrm{ps}$ e de acordo com a equação (5a), $\mathrm{L}_{\mathrm{D}}$ é da ordem de alguns poucos quilômetros e os efeitos de dispersão podem ser desprezados para propagação em trechos curtos de fibra $\left(\mathrm{L}_{\mathrm{D}}>>\mathrm{L}\right)$. Os outros parâmetros são escolhidos de forma que o efeito combinado do descasamento de velocidade de grupo (walk-off) e das não-linearidades (SPM e XPM) seja observado na propagação $\left(\mathrm{L}_{W}, \mathrm{~L}_{\mathrm{NL}}<<\mathrm{L}\right)$.

Nessa particular situação, a equação (1) é reescrita como um par de equaçóes acopladas, onde as derivadas temporais de segunda e terceira ordens são desprezadas.

$$
\begin{gathered}
\frac{\partial \mathrm{A}_{1}}{\partial \mathrm{z}}=\mathrm{j} \gamma_{1}\left(\left|\mathrm{~A}_{1}\right|^{2}+2\left|\mathrm{~A}_{2}\right|^{2}\right) \mathrm{A}_{1} \\
\frac{\partial \mathrm{A}_{2}}{\partial \mathrm{z}}+\mathrm{d} \frac{\partial \mathrm{A}_{2}}{\partial \mathrm{T}}=\mathrm{j} \gamma_{2}\left(\left|\mathrm{~A}_{2}\right|^{2}+2\left|\mathrm{~A}_{1}\right|^{2}\right) \mathrm{A}_{2}
\end{gathered}
$$

As equações acopladas (6) têm solução analítica apresentada em (AGRAWAL, 2006), (GODOY, 2006), que é convenientemente utilizada para validar os primeiros resultados numéricos obtidos.
As Figuras 2 a 4 mostram, para três situações distintas: (a) a evolução ao longo da fibra dos envelopes dos pulsos verde e laranja, que têm comportamentos de probe e pump, respectivamente e (b) o espectro do pulso verde na saída da fibra.

Na Figura 2, os pulsos verde e laranja são lançados simultaneamente $\left(\mathrm{T}_{\mathrm{d}}=0\right)$; na Figura 3, o pulso laranja é lançado com retardo inicial de $\mathrm{T}_{\mathrm{d}}=20$ ps em relação ao pulso verde e na Figura 4, o pulso laranja é lançado com retardo inicial de $\mathrm{T}_{\mathrm{d}}=40$ ps em relação ao pulso verde. Nas simulações foram usados os seguintes parâmetros: $\mathrm{P}_{2} / \mathrm{P}_{1}$ $=1000, \gamma_{1} \mathrm{P}_{2} \mathrm{~L}=40$ e $\mathrm{L}=4 \mathrm{~m}$.

Como pode ser observado nas Figuras 2a, 3a e 4a, o envelope (forma temporal) de cada pulso se mantém inalterado, pois os efeitos dispersivos são desprezados.

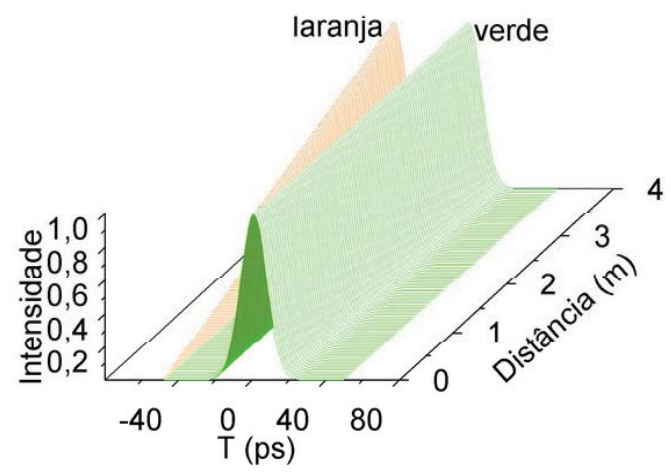

(a)

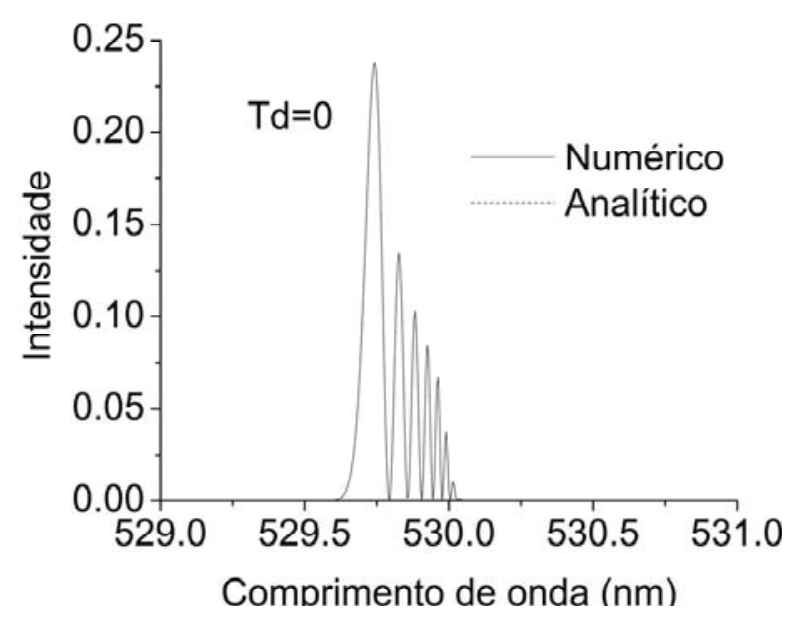

(b)

Figura 2: (a) Evolução dos envelopes dos pulsos verde (probe) e laranja (pump) ao longo da fibra e (b) espectro do pulso verde na saída da fibra: pulso laranja lançado simultaneamente $\left(\mathrm{T}_{\mathrm{d}}=0\right)$ ao pulso verde $\left(\mathrm{P}_{\text {laranja }} / \mathrm{P}_{\text {verde }}=1000, \gamma_{1} \mathrm{P}_{\text {laranja }} \mathrm{L}\right.$ $=40$ e $\mathrm{L}=4 \mathrm{~m}$ ). 


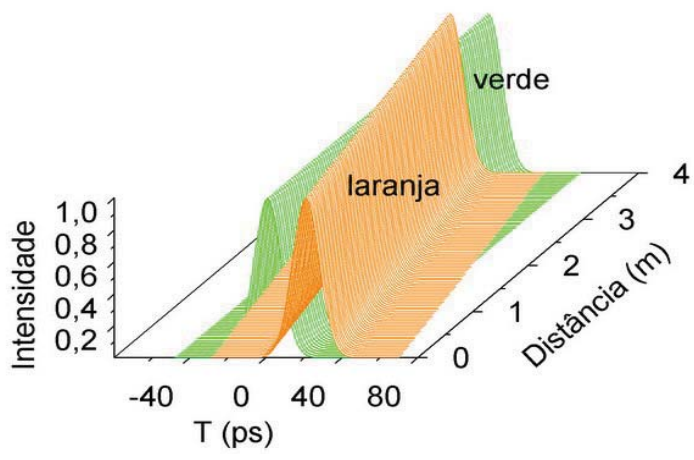

(a)

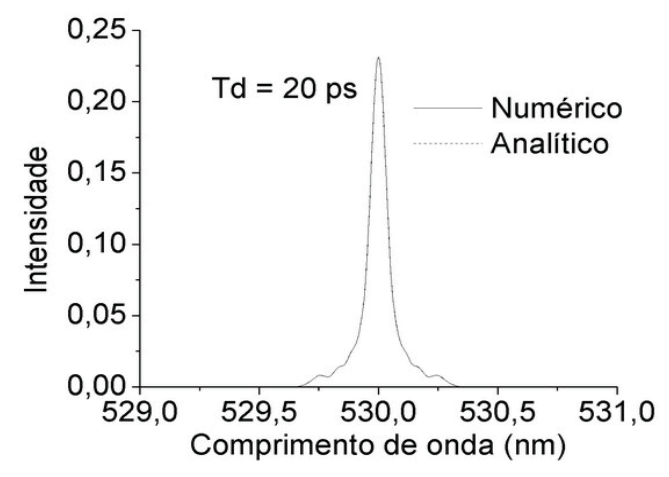

(b)

Figura 3: (a) Evolução dos envelopes dos pulsos verde (probe) e laranja (pump) ao longo da fibra e (b) espectro do pulso verde na saída da fibra: pulso laranja lançado $\mathrm{T}_{\mathrm{d}}=20$ ps após o pulso verde $\left(\mathrm{P}_{\text {laranja }} / \mathrm{P}_{\text {verde }}=1000, \gamma_{1} \mathrm{P}_{\text {laranja }} \mathrm{L}=40\right.$ e $\left.\mathrm{L}=4 \mathrm{~m}\right)$.

Nas Figuras 2b, 3b e 4b, os resultados analíticos (AGRAWAL, 2006), (GODOY, 2006) são incluídos e comparados com os numéricos agora obtidos, com excelente concordância.

Em cada uma das três situações estudadas, observa-se um comportamento distinto para o espectro do pulso verde na saída da fibra, dependendo do retardo inicial entre os pulsos. Tendo em vista que o pulso laranja (de maior comprimento de onda) se propaga mais rápido que o pulso verde, na situação da Figura 2, o pulso verde interage principalmente com a cauda do pulso laranja durante toda a propagação e, conseqüentemente, seu espectro se alarga e se desloca para a esquerda (comprimentos de onda mais curtos). Na situação ilustrada na Figura 3, o pulso laranja ultrapassa o pulso verde em $\mathrm{L} / 2$ e as interaçóes na frente $\mathrm{e}$ cauda do pulso verde praticamente se cancelam. Dessa forma, o espectro do pulso verde na saída da fibra se alarga simetricamente. Na Figura 4, observa-se que o pulso laranja só atinge o pulso verde na saída da fibra e, portanto, durante toda a propagação, o pulso verde interage basicamente com a frente do pulso laranja, tendo seu espectro alargado e deslocado para a direita (comprimentos de onda mais longos).

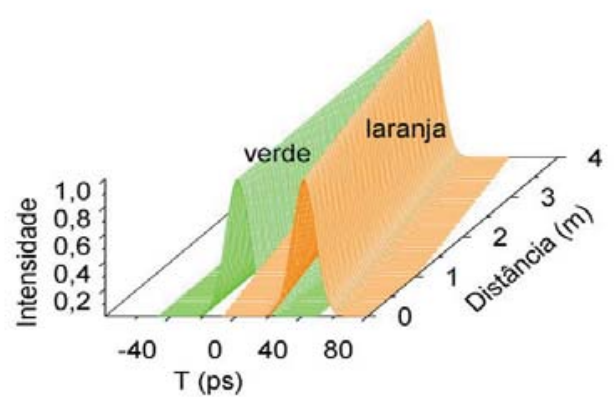

(a)

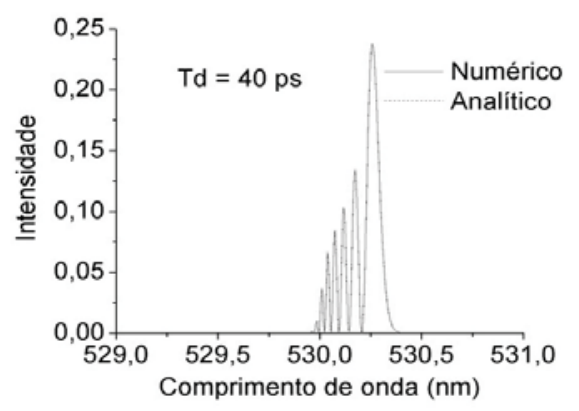

(b)

Figura 4: (a) Evolução dos envelopes dos pulsos verde (probe) e laranja (pump) ao longo da fibra e (b) espectro do pulso verde na saída da fibra: pulso laranja lançado $T_{d}=40$ ps após o pulso verde $\left(\mathrm{P}_{\text {laranja }} / \mathrm{P}_{\text {verde }}=1000, \gamma_{1} \mathrm{P}_{\text {laranja }} \mathrm{L}=40\right.$ e $\left.\mathrm{L}=4 \mathrm{~m}\right)$.

O alargamento espectral assimétrico observado é denominado deslocamento de freqüência induzido por XPM e é utilizado em diversas áreas da Comunicação Óptica (AGRAWAL; BALDECK; ALFANO, 1989), (AGRAWAL, 2005). O pulso laranja (pump), com potência de pico 1000 vezes maior que a do pulso verde (probe) sofre, fundamentalmente, o efeito de SPM e o chirp de freqüência (alargamento espectral) é quase simétrico. Nesta situação, o espectro do pulso laranja na saída da fibra é semelhante ao do pulso verde, $\operatorname{com} \mathrm{T}_{\mathrm{d}}=20 \mathrm{ps}$ (Figura 3b), motivo pelo qual não foi mostrado.

Clara e conclusivamente, os casos estudados mostram que o chirp induzido por XPM no pulso verde (probe) é afetado pelo descasamento de velo- 
cidade de grupo (walk-off) e depende criticamente do retardo inicial entre os pulsos.

Simulações considerando a propagação de quatro pulsos também foram realizadas e os resultados revelaram comportamentos semelhantes aos de dois pulsos. Observou-se que o envelope dos pulsos não se altera durante a propagação, enquanto que a assimetria do espectro de cada pulso depende de sua posição na seqüência de pulsos. Por exemplo, considerando que os pulsos têm potências iguais e são lançados simultaneamente na fibra, o espectro dos pulsos mais externos (pulsos 1 e 4) é mais assimétrico do que o dos pulsos internos (pulsos 2 e 3). A explicação para este fato é que o chirp induzido por XPM em cada um dos pulsos externos recebe a contribuição dos outros três pulsos. Por outro lado, para os pulsos internos (2 e 3), os chirps induzidos por seus pulsos vizinhos praticamente se cancelam. A contribuição para a assimetria do espectro desses pulsos é, portanto, menos acentuada, pois advém de um único pulso, o mais afastado.

\subsection{XPM NA PRESENÇA DE GVD}

$\mathrm{Na}$ discussão anterior, os efeitos da dispersão foram desprezados e o envelope dos pulsos se manteve inalterado, enquanto variaçóes nos correspondentes espectros foram observadas. Nesta seção, os efeitos conjuntos de GVD, SPM, XPM e walk-off são discutidos e, para tal, soluções numéricas completas da equação (1) são consideradas. Dois fenômenos distintos são estudados: quebra de onda óptica e geração de pulso a partir de onda CW.

\subsubsection{Quebra de onda óptica}

Uma configuração semelhante àquela estudada na Seção 3.1 é considerada, incluindo-se, agora, o efeito da dispersão (GVD). As alterações no envelope e espectro dos pulsos verde e laranja são analisadas no regime de dispersão normal e os resultados são comparados com os da seção anterior. Novamente, a configuração pump/probe é adotada. Os parâmetros de propagação são escolhidos de forma que: $\mathrm{L} / \mathrm{L}_{\mathrm{D}}=0,4, \mathrm{~L}_{\mathrm{D}} / \mathrm{L}_{\mathrm{W}}=10, \mathrm{~L}_{\mathrm{D}} /$ $\mathrm{L}_{\mathrm{NL}}=100$. Os pulsos têm largura $\mathrm{T}_{0}=5$ ps. Para $\mathrm{P}_{1} \ll \mathrm{P}_{2}=8 \mathrm{~W}$, o pulso verde (probe) sofre, principalmente, o efeito conjunto de GVD e XPM, enquanto que o pulso laranja ( $p$ pmp) sofre o efeito conjunto de GVD e SPM. Considera-se, ainda, que os pulsos verde e laranja são lançados simultaneamente na fibra $\left(T_{d}=0\right)$.

A Figura 5 mostra (a) a forma de onda (envelope) e (b) o espectro do pulso verde na saída da fibra para as condições de propagação anteriormente especificadas.

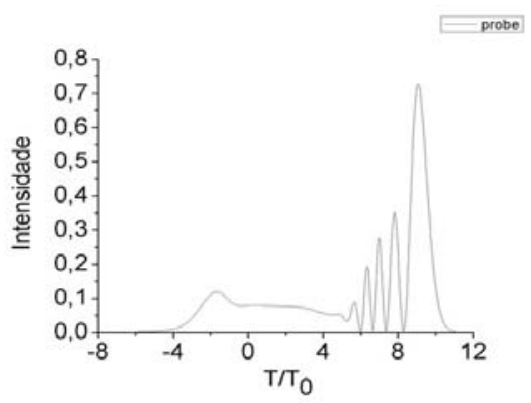

(a)

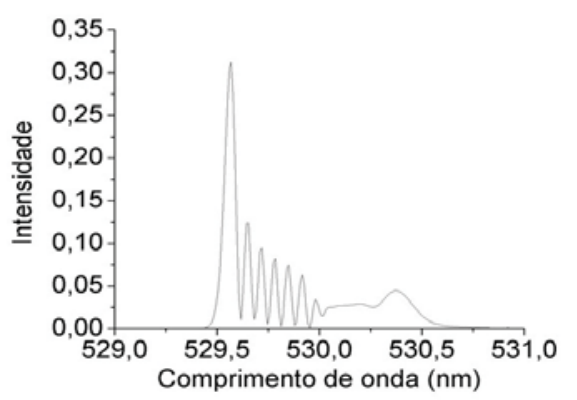

(b)

Figura 5: (a) Forma de onda (envelope) e (b) espectro do pulso verde (probe) na saída da fibra, sob o efeito conjunto de GVD, SPM, XPM e walk-off. $\left(\mathrm{L} / \mathrm{L}_{\mathrm{D}}=0,4, \mathrm{~L}_{\mathrm{D}} / \mathrm{L}_{\mathrm{W}}=10\right.$, $\left.\mathrm{L}_{\mathrm{D}} / \mathrm{L}_{\mathrm{NL}}=100\right)$.

A comparação do espectro do pulso verde (Figura 5b) com aquele obtido na ausência de GVD (Figura 2b), revela que o efeito da dispersão é reduzir a intensidade das oscilações espectrais causadas por XPM. Parte da energia do pulso é agora transportada por comprimentos de onda mais longos. O principal efeito da inclusão de GVD, no entanto, é notado no envelope do pulso verde, que se alarga e adquire uma estrutura bastante assimétrica, com rápidas oscilações na cauda. Se os comprimentos de onda dos pulsos verde e laranja forem trocados um pelo outro, as oscilações temporais aparecem na frente do pulso.

A Figura 6 considera as mesmas condições de propagação da Figura 5, analisando agora o pulso laranja. 


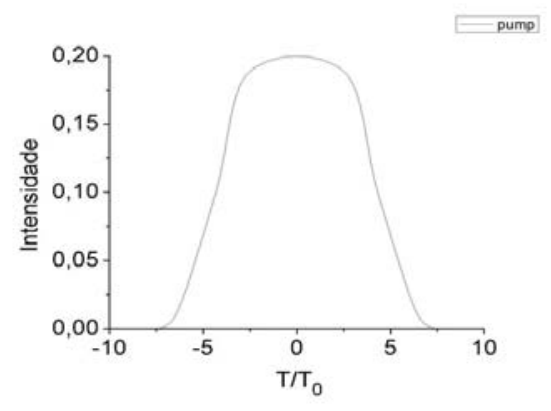

(a)

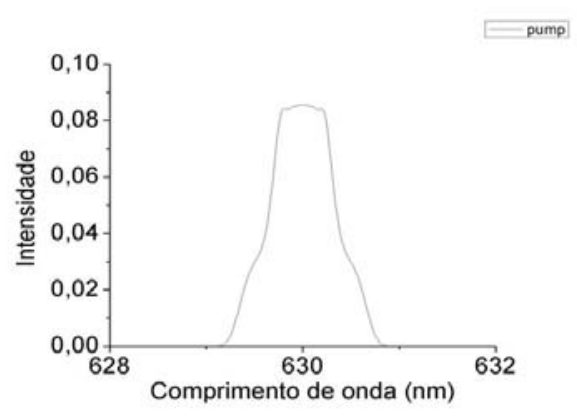

(b)

Figura 6: (a) Forma de onda (envelope) e (b) espectro do pulso laranja ( ( ump) na saída da fibra, sob o efeito conjunto de GVD, SPM, XPM e walk-off. $\left(\mathrm{L}_{\mathrm{L}} \mathrm{L}_{\mathrm{D}}=0,4, \mathrm{~L}_{\mathrm{D}} / \mathrm{L}_{\mathrm{W}}=10\right.$, $\left.\mathrm{L}_{\mathrm{D}} / \mathrm{L}_{\mathrm{NL}}=100\right)$.

Neste caso, o chirp induzido por SPM é mais significativo que o chirp induzido por XPM, pois o pulso laranja é bem mais intenso que o pulso verde.

Na Figura 6 é possível observar, com clareza, o conhecido fenômeno de quebra de onda óptica (optical wave breaking), onde o pulso assume um formato quase retangular, com suas bordas (frente e cauda) tão mais abruptas quanto mais o efeito de SPM domina sobre o de GVD (ANDERSON, 1993), (AGRAWAL, 2006).

A Figura 7 mostra, ainda, o espectro do pulso verde na saída da fibra, considerando, agora, que os pulsos verde e laranja são lançados na fibra com retardo inicial $T_{d}=20$ ps. Primeiramente, esta situação pode ser comparada com a da Figura 3b (ausência de GVD) e revela que o espectro do pulso verde deixa de ser simétrico, sofre alargamento e desloca-se para a direita (comprimentos de onda mais longos), uma conseqüência do chirp induzido pela dispersão, que havia sido desprezada na Seção 3.1.
Outra notável observação, a partir de uma comparação das Figuras 5b e 7, é que a estrutura oscilatória do espectro do pulso verde praticamente desaparece quando há retardo inicial entre os pulsos. Na situação representada na Figura 7, o envelope do pulso sofre compressão nos estágios iniciais da propagação, para então se alargar progressivamente. A compressão de pulsos representa uma importante aplicação das não-linearidades ópticas na obtenção de pulsos ultracurtos (GUO; ZHOU, 2006).

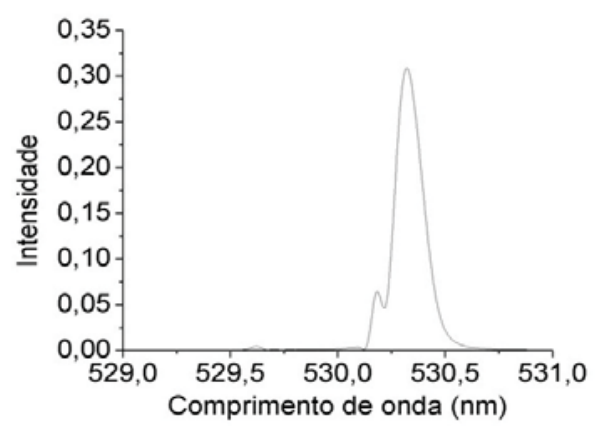

Figura 7: Espectro do pulso verde na saída da fibra, sob o efeito conjunto de GVD, SPM, XPM e walk-off. O pulso laranja foi lançado com retardo de 20 ps; demais parâmetros como na Figura 5.

\subsubsection{Geração de pulso a partir de onda CW}

Para completar a análise, a importante técnica de geração de trens de pulsos escuros a partir de onda CW é descrita. Para tal, considera-se a interação de um sinal $\mathrm{CW}$ de baixa intensidade $(1565 \mathrm{~nm}, 2 \mathrm{~mW})$ com um pulso gaussiano intenso $\left(1557,1 \mathrm{~nm}, 1,3 \mathrm{~W}, \mathrm{~T}_{\mathrm{FWHM}}=31,7 \mathrm{ps}\right) \mathrm{em} \mathrm{um}$ trecho curto $(1,37 \mathrm{~km})$ de fibra DSF $\left(\lambda_{0}=1570\right.$ $\mathrm{nm}, \gamma=3(\mathrm{~W} . \mathrm{km})^{-1}, \beta_{2}\left(\lambda_{\mathrm{s}}\right)=0,9 \mathrm{ps}^{2} / \mathrm{km}, \beta_{2}\left(\lambda_{\mathrm{p}}\right)=$ $\left.2,3 \mathrm{ps}^{2} / \mathrm{km}\right)$. A propagação ocorre, portanto, no regime de dispersão normal. Os parâmetros usados nas simulações são os mesmos do experimento descrito em (CAO; LI; CHAN, 1999), de maneira a validar os resultados numéricos obtidos.

A Figura 8 mostra a evolução (a) do pulso gaussiano e (b) do sinal CW. Para o pulso gaussiano, $\mathrm{L}_{\mathrm{D}} \approx 160 \mathrm{~km}$, de acordo com a Equação (5a), e o alargamento temporal do envelope do pulso é, portanto, quase imperceptível. O alargamento espectral induzido no pulso gaussiano ( $p$ ump) não está mostrado, mas é simétrico e devido fundamentalmente ao efeito de SPM, uma vez que o sinal CW é de baixa intensidade. 


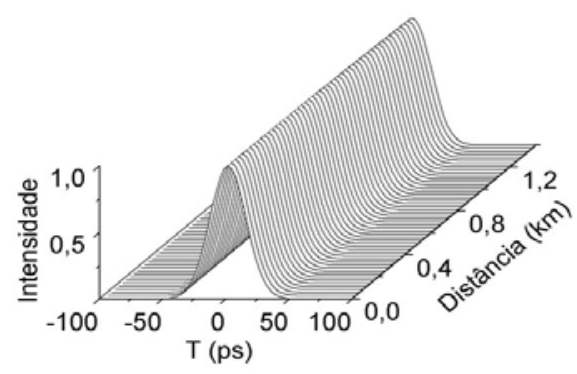

(a)

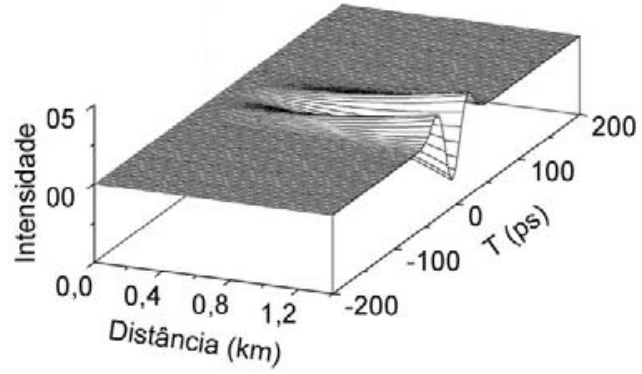

(b)

Figura 8: Evolução (a) de um pulso gaussiano $(1557,1 \mathrm{~nm}$, $\left.1,3 \mathrm{~W}, \mathrm{~T}_{\mathrm{FWHM}}=31,7 \mathrm{ps}\right)$ e (b) de um sinal CW (1565 nm, 2 $\mathrm{mW})$ em um trecho curto $(1,37 \mathrm{~km})$ de fibra DSF.

Por outro lado, durante toda a propagação, o sinal CW tem sua fase intensamente modulada pelo pulso gaussiano (efeito XPM). Como resultado, as componentes de freqüência mais baixas posicionam-se na frente do pulso e as mais altas, na cauda do pulso. Esse agrupamento de freqüências coincide com aquele provocado pela dispersão no regime de dispersão normal, o que acarreta uma modulação em amplitude e constitui uma técnica para a geração de trens de pulsos escuros. Como pode ser claramente observado na Figura 8b, um pulso escuro se forma em torno de $0,4 \mathrm{~km}$, o que está em perfeita concordância com os resultados experimentais descritos em (CAO; LI; CHAN, 1999). O alargamento espectral induzido no sinal $\mathrm{CW}$ é apenas ligeiramente assimétrico, uma vez que o descasamento de velocidade de grupo é bastante pequeno. Vale a pena ressaltar que a geração de pulsos escuros depende fortemente do efeito conjunto de XPM e GVD no regime de dispersão normal.

Em uma outra situação interessante, derivada da anterior, analisa-se a interação entre um sinal CW de baixa intensidade e dois pulsos gaussianos intensos $\left(1535 \mathrm{~nm}, \mathrm{~T}_{\mathrm{FWHM}}=8 \mathrm{ps}\right) \mathrm{em} \mathrm{um}$ trecho de $5 \mathrm{~km}$ de fibra DSF $(\overline{\overline{0}}=1542 \mathrm{~nm},=3$ $\left.(\mathrm{W} . \mathrm{km})^{-1}\right)$. O comprimento de onda do sinal CW varia de 1538 a $1558 \mathrm{~nm}$, para que o comportamento do sinal $\mathrm{CW}$ nos regimes de dispersão normal e anômala seja observado. Os parâmetros usados nas simulações são os mesmos do experimento descrito em (ÔHLÉN; OLSSON; BLUMENTHAL, 2000), de maneira a validar os resultados numéricos obtidos.

A Figura 9 mostra a evolução do sinal CW nos regimes de dispersão (a) normal (1540nm) e (b) anômala (1550nm).

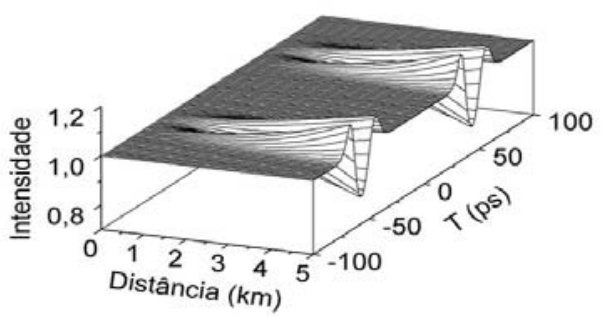

(a)

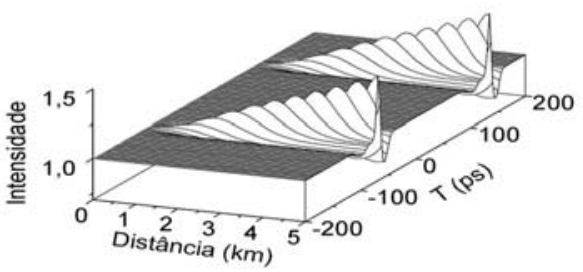

(b)

Figura 9: Evolução do sinal $\mathrm{CW}$ nos regimes de dispersão (a) normal $(1540 \mathrm{~nm})$ e (b) anômala $(1550 \mathrm{~nm})$, que interage com dois pulsos gaussianos intensos $\left(1535 \mathrm{~nm}, \mathrm{~T}_{\mathrm{FWHM}}=\right.$ 8 ps) em um trecho de $5 \mathrm{~km}$ de fibra DSF.

Como esperado, o comportamento no regime de dispersão normal é semelhante ao observado na Figura 8b, com a formação de um trem de dois pulsos escuros. Já no regime de dispersão anômala, os chirps de freqüência induzidos por GVD e XPM são contrários, propiciando a formação de pulsos claros. Esse mecanismo é utilizado para a conversão de comprimento de onda em redes ópticas (ÔHLÉN; OLSSON; BLUMENTHAL, 2000). 


\section{CONCLUSÕES}

$\mathrm{O}$ presente trabalho investigou o impacto dos efeitos de dispersão de velocidade de grupo (GVD), não-linearidades (SPM e XPM) e descasamento de velocidade de grupo na propagação de pulsos em fibras ópticas. As resultantes alteraçóes na forma de onda (envelope) e no espectro de pulsos co-propagantes em fibra óptica monomodo padrão (SMF) e de dispersão deslocada (DSF) podem afetar negativamente o desempenho de sistemas DWDM. O modelo numérico desenvolvido é baseado na solução de equações NSLE acopladas e permite uma adequada avaliação destes efeitos. A configuração pump/probe foi utilizada para isolar os efeitos de XPM e SPM. Os efeitos de GVD foram considerados e ilustrados em dois casos separados.

Os resultados obtidos mostraram que, na ausência de dispersão, o envelope dos pulsos se mantém inalterado, enquanto o espectro do probe se alarga, com estrutura assimétrica (chirp induzido por XPM), fortemente dependente do retardo temporal inicial entre os pulsos. A inclusão dos efeitos dispersivos mostrou que o envelope e espectro dos pulsos são alterados e permitiu observar o fenômeno de quebra de onda óptica, assim como a possibilidade de se utilizar o efeito de XPM para comprimir pulsos de baixa intensidade.

Em uma outra aplicação do simulador numérico, observou-se a geração de pulsos escuros e claros em fibra DSF, a partir de sinal CW de baixa intensidade, considerando os regimes de dispersão normal e anômala, respectivamente. Esta é uma aplicação de grande utilidade no contexto de redes ópticas de alta velocidade, pois permite o projeto de conversores de comprimento de onda.

As simulaçôes realizadas confirmam a necessidade de judiciosa análise/avaliação de efeitos não-lineares no projeto de sistemas ópticos DWDM. Neste sentido, o modelo numérico desenvolvido mostrou-se confiável, robusto e versátil. Em todos os casos analisados, os resultados estão em excelente concordância com os disponíveis na literatura.

\section{REFERÊNCIAS}

AGRAWAL, G. P. Lightwave Technology: Telecommunication Systems, John Wiley \& Sons, Inc., Hoboken, 2005.
Nonlinear Fiber Optics ( $4^{\text {th }}$ ed.),

Academic Press, San Diego, USA, 2006.

AGRAWAL, G.P., BALDECK P.L., ALFANO R.R. Temporal and spectral effects of cross-phase modulation on copropagating ultrashort pulses in optical fibers, Phys. Rev. , v. A 39, n. 9, p. 50635072, 1989.

ANDERSON, D. et al. Wave-breaking-free pulses in nonlinear-optical fibers, Journal Opt. Soc. Am. B v. 10, n. 7, p. 1185-1190, 1993.

CAO,W., LI, S., K. CHAN, K. Generation of dark pulse trains from continuous-wave light using cross-phase modulation in optical fibers, Applied Physics Letters, v. 74, n. 4 , p. 510-512, 1999.

GODOY, D.S. Simulador WDM em Fibra Óptica Dispersiva, Não-Linear e com Perdas, Dissertação de Mestrado apresentada ao Departamento de Engenharia de Telecomunicações da Universidade Federal Fluminense, Niterói, 2006.

GUO, L., ZHOU, C. Spectral Narrowing of negatively chirped femtosecond pulse by cross-phase modulation in a single-mode optical fiber, Optics Communication, v. 260, p. 140-143, 2006.

HARBOE, P.B., GODOY, D.S., SOUZA, J.R. Simulador WDM em Fibra Óptica Dispersiva, Não-Linear e com Perdas, Anais MOMAG 2006: $12^{\circ}$ SBMO - Simpósio Brasileiro de Microondas e Optoeletrônica e $7^{\circ} \mathrm{CBMag}$ - Congresso Brasileiro de Eletromagnetismo, Belo Horizonte - MG, 2006.

HARBOE, P.B., SILVA, E., SOUZA, J.R., Simulador WDM para Análise das Penalidades Impostas por FWM em Fibras G.652, G.653 e G.655, Anais MOMAG 2008: $13^{\circ}$ SBMO - Simpósio Brasileiro de Microondas e Optoeletrônica e $8^{\circ}$ CBMag - Congresso Brasileiro de Eletromagnetismo, Florianópolis - SC, 2008a.

FWM em Sistemas DWDM: Espaçamento Não Uniforme versus Espaçamento Uniforme, Anais SBrT'08: XXVI Simpósio Brasileiro de Telecomunicações, Rio de Janeiro - RJ, 2008b. 
Analysis of FWM Penalties in DWDM Systems Based on G.652, G.653, and G.655 Optical Fibers, submetido ao MWON 2008 - Fifth International Conference on Mobile, Wireless and Optical Communications Networks, a ser realizado em Bangkok - Tailândia, dec. 2008c.

LEIBRICH, J., ROSENKRANZ, W. Efficient Numerical Simulation of Multichannel WDM Transmission Systems Limited by XPM, IEEE Photonics Technology Letters, v. 15, n. 3, p. 395397, 2003.

ÔHLÉN, P., OLSSON, B., BLUMENTHAL, D.J. Wavelength Dependence and Power Requirements of a Wavelength Converter Based on XPM in a Dispersion-Shifted Optical Fiber, IEEE Photonics Technology Letters, v. 12, n. 5, p. 522-524, 2000.

ROUDAS, I. et al. Wavelength-Domain Simulation of Multiwavelength Optical Networks, IEEE
Journal of Selected Topics in Quantum Electronics, v. 6, n. 2, p. 348-362, 2000.

SILVA, E. Simulador WDM para Análise das Penalidades Impostas por Mistura de Quatro Ondas (FWM) em Fibras Ópticas Monomodo SMF, DSF e NZDSF, Dissertação de Mestrado apresentada ao Departamento de Engenharia de Telecomunicações da Universidade Federal Fluminense, Niterói, 2008.

SOUZA, J.R., HARBOE, P.B. Assessment of Higher-Order Exponential Operators for the Simulation of High-Capacity Optical Communication Systems by the Split-Step Fourier Method, Journal of Microwaves and Optoelectronics, v. 3, n. 2, p. 9-22, 2003.

WU, M., WAY, W.I. Fiber Nonlinearity Limitations in Ultra-Dense WDM Systems, Journal of Lightwave Technology, v. 22, n. 6, p. 1483-1497, 2004. 